\title{
Clinical symptoms, diagnosis and outcome of septic encephalopathy
}

\author{
Ahmed Ragab Zein ${ }^{1 *}$, Ayat Bagi Algassab ${ }^{2}$, Mada Salem Almatrafi ${ }^{3}$, Hawazen Atef Kamal ${ }^{4}$, \\ Abdulilah Mokhtar Alshenghiti ${ }^{5}$, Bandar Merzen Alqarni ${ }^{6}$, Ali Jaber Ashoori ${ }^{7}$, \\ Faisal Abdulsalam Albloushi ${ }^{8}$, Mahmood Abdulla Alshaban ${ }^{7}$, Asma Sulaiman Alshahrani', \\ Mohammed Emran Dawoud ${ }^{10}$
}

\author{
${ }^{1}$ Department of Intensive Care Unit, King Fahad General Hospital, Jeddah, Saudi Arabia \\ ${ }^{2}$ Department of Emergency Medicine, Dammam Medical Complex, Dammam, Saudi Arabia \\ ${ }^{3}$ Department of Intensive Care Unit, King Abdullah Medical Complex, Jeddah, Saudi Arabia \\ ${ }^{4}$ Department of Neurosurgery, Al Noor Specialist Hospital, Mecca, Saudi Arabia \\ ${ }^{5}$ Department of General Surgery, Diriyah Hospital, Riyadh, Saudi Arabia \\ ${ }^{6}$ Department of Family Medicine, King Fahad Armed Forces Hospital, Jeddah, Saudi Arabia \\ ${ }^{7}$ College of Medicine, Xi'an Jiaotong University, Xi'an, China \\ ${ }^{8}$ College of Medicine, Jinan University, Guangzhou, China \\ ${ }^{9}$ College of Medicine, King Khalid University, Abha, Saudi Arabia \\ ${ }^{10}$ Primary Health Care, Ministry of Health, Kuwait City, Kuwait
}

Received: 25 December 2021

Revised: 11 January 2022

Accepted: 12 January 2022

\section{*Correspondence:}

Dr. Ahmed Ragab Zein,

E-mail: ahmadragab@gmail.com

Copyright: (C) the author(s), publisher and licensee Medip Academy. This is an open-access article distributed under the terms of the Creative Commons Attribution Non-Commercial License, which permits unrestricted non-commercial use, distribution, and reproduction in any medium, provided the original work is properly cited.

\begin{abstract}
Septic encephalopathy (SE) is the most common complication of sepsis and a major cause of mortality and morbidity worldwide. It occurs in around two third of patients admitted with sepsis in the intensive care units. The objective of this review is to discuss clinical symptoms, diagnosis and outcomes in patients with SE. Robust research was conducted from online databases such as Google Scholar and Cochrane. Studies from the last ten years were included in our review. The pathophysiology of SE is highly dynamic where a complex sequence of events occurs as a host response to an extracranial infection. SE presents a myriad of symptoms ranging from an altered mental state with confusion, disorientation to a more severe presentation with delirium and impaired consciousness. Arriving to a diagnosis of SE requires a rigorous systemic and neurological investigation. Modalities such as neuromonitoring, neuroimaging, clinical biomarkers and mental assessment scales offer limited diagnostic value but can be utilized to co-relate clinical symptoms. It is associated with adverse outcomes such as a high rate of 28-day and 180-day mortality. The quality of life is poor in surviving patients. There is a dearth of original research on SE in the Arab region and worldwide. Major research, both as prospective and retrospective studies is essential to achieve improved management of SE. Therapeutic approaches should be tailored towards modulating pathophysiological processes of sepsis. Follow up studies based on 180 day follow ups should be used to study patient quality of life and outcomes.
\end{abstract}

Keywords: Septic, Encephalopathy, Clinical, Symptoms, Outcome, Diagnosis

\section{INTRODUCTION}

Sepsis is one of the leading causes of admissions in the intensive care unit (ICU) and one of the most frequent causes of mortality. ${ }^{1-3}$ The third international consensus definitions for sepsis and septic shock (Sepsis-3) have evaluated and revised outdated definitions. Sepsis is defined as a syndrome of organ dysfunction due to a dysregulated response by the host to an infection, and is deemed life-threatening. ${ }^{4}$ Septic shock is a subset of 
sepsis, where the host's consequential immune-mediated, metabolic response to infection substantially contribute towards mortality. Septic Shock typically requires vasopressor treatment to maintain an average arterial pressure during one cardiac cycle (both systole and diastole) to $65 \mathrm{mmHg}$ or higher, and lactate serum levels greater than 2 , despite continuous fluid resuscitation. ${ }^{4}$

Septic encephalopathy (SE) is a diffuse cerebral dysfunction which occurs due to the system's inflammatory response to sepsis. It occurs in the absence of a primary central nervous system (CNS) insult or infection. $^{3}$ There is major ambiguity regarding the appropriate term SE and a universally accepted definition is absent. ${ }^{3}$ Therefore, SE is used as an umbrella term for a wide range of neurological signs and symptoms that manifest in the event of sepsis. Other terms such as sepsis-associated encephalopathy (SAE), sepsis associated brain dysfunction (SABD) and Sepsis induced brain dysfunction (SIBD) are also used in multiple studies.

SE is the most common complication of sepsis and develops in $70 \%$ of septic patients. ${ }^{1,2,5}$ It is the most common type of encephalopathy to develop among ICU patients. ${ }^{1,6} \mathrm{SE}$ is associated with cognitive impairment and disability, in surviving patients. $^{7}$ Long term consequences, such as poor quality of life, anxiety, depression develop in $62 \%$ of patients suffering from SE. ${ }^{1}$ The aim of this review is to extensively discuss clinical symptoms, outcomes, and diagnosis of SE. For this review, SE will be used interchangeably with SAE.

\section{LITERATURE REVIEW}

This literature review is based on an extensive literature search in Medline, Cochrane, and EMBASE databases which was performed on $07^{\text {th }}$ December 2021 using the medical subject headings (MeSH) or a combination of all possible related terms, according to the database. Only papers within the last ten years were included. To avoid missing potential studies, a further manual search for papers was done through Google Scholar.

\section{DISCUSSION}

The pathophysiology of SE is highly dynamic where complex mechanisms with a cascade of events, neurodegenerative and neurovascular are occurring simultaneously. A few proposed mechanisms are inflammatory mediators and the complement cascade, wherein positive feedback mediated imbalance between pro inflammatory cytokines such as tumor necrosis factor alpha (TNF- $\alpha$ ) and gamma interferon, and antiinflammatory cytokines such as Interleukins. Sustained neuroinflammation leads to SE. Other speculations are endothelial dysfunction and associated damage to the blood brain barrier (BBB). An increased influx of inflammatory cytokines through the compromised BBB triggers microcytic and glial activation. ${ }^{8}$

\section{Clinical symptoms}

An altered mental status and impaired cognition with an extracranial infection is a hallmark feature of SE. ${ }^{9,10}$ Clinical symptoms of encephalopathy affect nearly $70 \%$ of septic patients. ${ }^{5}$ During the early stages, patients exhibit milder symptoms such as confusion, inattentiveness, irritability, and disorientation. Later stages of SE constitute more severe forms of SE such as delirium, impaired cognition, personality changes or agitation and can be observed in $82 \%$ of mechanically ventilated patients. ${ }^{5,9} \mathrm{SE}$ can also manifest as hypersomnolence, stupor and coma in the event of multi organ dysfunction. ${ }^{9}$ Often agitation and somnolence can take place in succession. ${ }^{8}$ Motor symptoms such as tremors, paratonic rigidity, asterixis, and even seizures both focal and generalized as observed in other encephalopathies are less frequent. ${ }^{5,8,11,12}$ Symptoms of impaired consciousness, and cognition are out of proportion to sedatives. ${ }^{13}$ Long term consequences such as poor quality of life, anxiety, depression, dementia, and even post-traumatic stress disorder is present in up to $62 \%$ of patients. ${ }^{1}$ Meanwhile, symptoms of cognitive impairment are present in $45 \%$ of patients up to 1 year after discharge. ${ }^{5}$

\section{Diagnosis}

A comprehensive neurological examination is the ideal way to screen for SE. ${ }^{1} \mathrm{SE}$ is entirely a diagnosis of exclusion. Therefore, an extensive neurological examination, to rule out an underlying CNS insult or pathology must be conducted. Afterwards, a robust systemic investigation including a complete hematological and chemical panel, to rule out other possible etiologies of the encephalopathy and a search for the focus of infection must be ensued..$^{6,13}$

Although various biomarkers are available, there is no ideal biomarker to diagnose or predict the probability or duration of SAE. ${ }^{14} \mathrm{~N}$-Terminal propeptide of CNP (NTproCNP) is a biomarker for endothelial dysfunction, S100 beta is a biomarker for glial injury and neuron specific enolase is a biomarker for neuronal injury. ${ }^{6,12} \mathrm{~A}$ systematic review on biomarkers of brain injury, was conducted in Brazil in 2013. Out of 9 studies, 7 were identified with raised concentrations of S100 beta, of which 2 studies did not find an association, and 1 study where no co-relation was observed. ${ }^{8}$ An increased level of these biomarkers can indicate severity, however, as their sensitivity and specificity are low, they offer limited diagnostic value..$^{9,12,13}$

Electroencephalogram, (EEG) is non-invasive test that records the electrical activity of the brain to diagnose brain dysfunctions. In a study by Young et al 69 patients with encephalopathy were studied. Five different wave form patterns co-relating to the severity of SE were observed. The most severe wave form, which is suppression or burst suppression or was found in $67 \% .5,9$ 
Oddo et al observed epileptiform discharges and seizure activity in $22 \%$ of patients with sepsis, whereas in two thirds the electroencephalographic findings did not corelate clinically. The EEG findings were positively corelated with multi-organ dysfunction and mortality. ${ }^{5}$ Although EEGs are highly sensitive, they are not specific and can exhibit similar findings in other encephalopathies. ${ }^{1}$

Clinical scales such as the confusion assessment method for the ICU (CAM-ICU) for non-intubated, and adaption to the intensive care environment (ATICE) help in a mental assessment of septic patients to assess comprehension. ${ }^{5} \mathrm{CAM}-\mathrm{ICU}$ is the most common scale to assess objective assessment. ${ }^{1}$ However, it shows sensitivity of $47 \%$ yet it's specificity is $98 \%{ }^{1,9}$ As these scales have limited value in sedated patients, thus Glasgow coma scale (GCS), the Richmond agitationagitation scale (RASS), the full outline of unresponsiveness (FOUR) for intubated patients can be used (I, III and VII). Despite multiple screening tools available, none are specific for SE. . $^{3,8,13}$

Imaging techniques such as MRI, CT Scan have been suggested in patients with SE, however, their usefulness is rather limited. ${ }^{1} \mathrm{MRI}$ is more sensitive in detecting nonstructural changes such as leukoencephalopathy and vasogenic edema. ${ }^{1}$ Cerebral ischemia is detected in $30 \%$ patients with sepsis and focal neurological deficits. ${ }^{3}$ According to a study, delirium and worsening of cognitive impairment for over one year is associated with lower brain volumes on MRI. ${ }^{1}$ Posterior reversible leukoencephalopathy Syndrome is also a common MRI finding in patients with SE (PRES). ${ }^{9,12}$

Features of SE such as developing early during sepsis, preceding organ dysfunction, in combination with the effect of sedatives, mechanical ventilation, and various presentations of SE make it a diagnostic challenge. ${ }^{1,5,8,13}$ Symptoms of SE such as weakness, anorexia, difficulty in concentration precede single or multi organ dysfunction which can be overlooked as constitutional symptoms and an overall sense of being unwell. ${ }^{13}$

\section{Outcome}

A retrospective report studying the adverse outcomes in patients with SE was conducted in Hangzhou, China in 2020. The primary outcomes of 28-day mortality and length of hospital stay were measured. A total of 291 patients were included in the study and were further categorized into SAE (127) and non-SAE (167) groups. The results showed 28-day mortality was considerably higher in patients with SAE $(42.5 \%)$ as compared to nonSAE groups $(12.8 \%)$ with a $\mathrm{p}<0.01$. A Kaplan Meier survival analysis confirmed the poor outcome in 28-day survival However, no significant differences were observed in hospital LOS in both groups. ${ }^{15}$ Another single-center retrospective study in Changsha, China was conducted between January 2015 to June 2016. After retrospective screening, 175 patients were included in the study and were divided into groups with SAE and without SAE groups. The 28-day mortality rate and 180-day mortality rate was analyzed. In addition, survival outcomes, assessment of quality of life and risk factor for death was also studied based on a180 day follow up. The study revealed the risk of death was significantly higher in patients with SAE as opposed to the non-SAE group. ( $R R=2.868 ; 95 \%$ confidence interval [CI]: 1.730-4.754; $\mathrm{p}<0.001)$. A higher rate of both 28-day mortality $(45.95 \%)$ and 180 -day mortality $(55.41 \%)$ the SAE group using a multivariate stepwise cox regression analysis, was also shown. Although patients with sepsis have a poor quality of life, with daily activities and pain, there was no significant difference in the quality of life among both groups. This study also did not show any significant difference in length of hospital stay between both groups $(\mathrm{p}=0.349){ }^{16}$

The major limitation of this review is a gap in literature due to a lack of prospective studies. Furthermore, retrospective studies are also very limited. There is a severe scarcity on studies conducted on SE in Saudi Arabia, and the regional gulf countries. After an extensive search, only one literature review on SE was retrieved. At present it is strongly recommended that more prospective, single-center, and multi-center studies should be conducted on SE particularly in the gulf region. Researchers should also take a keen interest in retrospective analyses of patients admitted with SE. Long term follow up on patients admitted with SE is also essential.

\section{CONCLUSION}

SE is the most common complication found in patients admitted with sepsis and is associated with high mortality, morbidity, and a poor quality of life in surviving patients. A unanimous, universally acknowledged definition is warranted. Arriving to a diagnosis of SE requires a thorough neurological and systemic examination and is purely a diagnosis of exclusion. Imaging modalities and laboratory investigations offer limited insight to the diagnosis. The incidence of $\mathrm{SE}$ can be reduced by preventing and managing sepsis. A better understanding of the pathophysiology with experimental models can revolutionize therapeutic approaches. Further research with both prospective and retrospective studies is essential to bridge the gap in literature.

\section{Funding: No funding sources \\ Conflict of interest: None declared \\ Ethical approval: Not required}

\section{REFERENCES}

1. Czempik PF, Pluta MP, Krzych ŁJ. Sepsis-associated brain dysfunction: a review of current literature. Int $\mathbf{J}$ Environmental Res Public Health. 2020;17(16):5852. 
2. Dal-Pizzol F, Tomasi CD, Ritter C. Septic encephalopathy: does inflammation drive the brain crazy? Braz J Psychiatry. 2014;36:251-8.

3. Robba C, Crippa IA, Taccone FS. Septic Encephalopathy. Curr Neurol Neurosci Rep. 2018;18(12):82.

4. Singer M, Deutschman CS, Seymour CW. The third international consensus definitions for sepsis and septic shock (Sepsis-3). JAMA. 2016;315(8):801-10.

5. Ziaja M. Septic encephalopathy. Curr Neurol Neurosci Rep. 2013;13(10):383.

6. M Ringer T, Axer H, FM Romeike B. Neurological sequelae of sepsis: I) Septic encephalopathy. Open Cri Care Med J. 2011;4(1).

7. Zenaide PV, Gusmao-Flores D. Biomarkers in septic encephalopathy: a systematic review of clinical studies. Revista Brasileira de terapia intensiva. 2013;25:56-62.

8. Adam N, Kandelman S, Mantz J, Chrétien F, Sharshar T. Sepsis-induced brain dysfunction. Expert Review Anti-Infective Therapy. 2013;11(2):211-21.

9. Chaudhry N, Duggal AK. Sepsis associated encephalopathy. Adv Med. 2014;2014.

10. Zampieri FG, Park M, Machado FS, Azevedo LCP. Sepsis-associated encephalopathy: not just delirium. Clinics. 2011;66:1825-31.
11. Imamura $\mathrm{Y}$, Hori $\mathrm{S}$, Matsumoto N. Septic encephalopathy. Encephalitis Encephalomyelitis Encephalopathies. 2013;8:163-76.

12. Mazeraud A, Righy C, Bouchereau E, Benghanem S, Bozza FA, Sharshar T. Septic-associated encephalopathy: a comprehensive review. Neurotherapeutics. 2020;17(2):392-403.

13. Gofton TE, Young GB. Sepsis-associated encephalopathy. Nature Rev Neurol. 2012;8(10):55766.

14. Tauber SC, Eiffert H, Brück W, Nau R. Septic encephalopathy and septic encephalitis. Expert Review Anti-infective Therapy. 2017;15(2):121-32.

15. Chen J, Shi X, Diao M. A retrospective study of sepsis-associated encephalopathy: epidemiology, clinical features and adverse outcomes. BMC Emergency Med. 2020;20(1):1-7.

16. Feng Q, Ai Y-H, Gong H. Characterization of sepsis and sepsis-associated encephalopathy. J Intensive Care Med. 2019;34(11-12):938-45.

Cite this article as: Zein AR, Algassab AB, Almatrafi MS, Kamal HA, Alshenghiti AM, Alqarni $\mathrm{BM}$ et al. Clinical symptoms, diagnosis and outcome of septic encephalopathy. Int J Community Med Public Health 2022;9:943-6. 